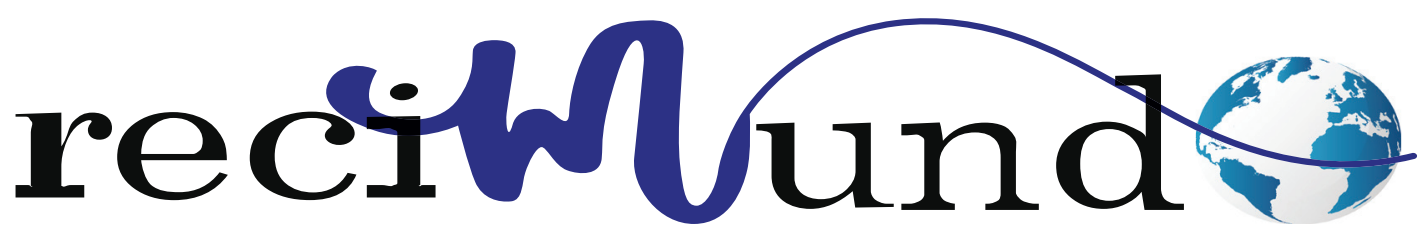

Revista Científica Mundo de la Investigación y el Conocimiento

DOI: 10.26820/recimundo/5.(2).abril.2021.119-127

URL: https://recimundo.com/index.php/es/article/view/1046

EDITORIAL: Saberes del Conocimiento

REVISTA: RECIMUNDO

ISSN: 2588-073X

TIPO DE INVESTIGACIÓN: Artículo de revisión

Código UNESCO: 32 Ciencias Médicas

PAGINAS: $119-127$

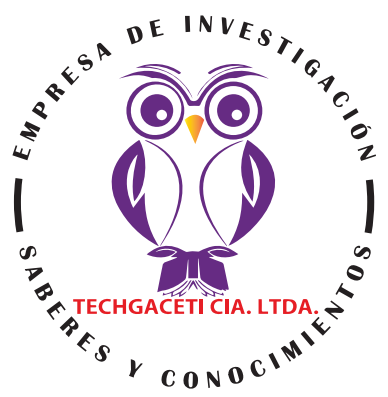

\title{
Valor predictivo entre Mechanical Power y Driving Pressure
}

Predictive value between Mechanical Power and Driving Pressure

Valor preditivo entre a força mecânica e a pressão motriz

Ariana Tatiana Choez Quimis'; Leonardo Xavier Quiroz Moncerrad²; Carlos Andrés Hidalgo Bermúdez;

Carmen Leonor González Plúa ${ }^{4}$

RECIBIDO: 15/01/2021 ACEPTADO: 20/03/2021 PUBLICADO: 01/04/2021

1. Médico de la Universidad de Guayaquil; Investigador Independiente; Guayaquil, Ecuador; aripet_czqs@hotmail.com; https://orcid.org/0000-0003-0297-3861

2. Médico de la Universidad de Guayaquil; Investigador Independiente; Guayaquil, Ecuador; Ixquiroz1988@hotmail.com; https://orcid.org/0000-0002-7728-529X

3. Médico de la Universidad Particular de Especialidades Espíritu Santo; Investigador Independiente; Guayaquil, Ecuador; carloshidalgo_92@hotmail.com; (iD https://orcid.org/0000-0002-8565-9407

4. Médico de la Universidad de Guayaquil; Investigador Independiente; Guayaquil, Ecuador; carmigp@gmail.com; (DD https:// orcid.org/0000-0003-3729-0104

CORRESPONDENCIA

Ariana Tatiana Choez Quimis

aripet_czqs@hotmail.com

Guayaquil, Ecuador

() RECIMUNDO; Editorial Saberes del Conocimiento, 2021 


\section{RESUMEN}

Tal como cualquier intervención farmacológica, la ventilación mecánica (VM) debe ser titulada dentro de una ventana terapéutica con el fin de que alcance el objetivo por el cual se inició y al mismo tiempo se limiten los efectos adversos de su empleo. Se ha propuesto que en "pacientes sin síndrome de distrés respiratorio agudo (SDRA) que son sometidos a ventilación mecánica, el driving pressure y el poder mecánico, son factores de riesgo para un aumento en la mortalidad y el desarrollo de SDRA. El objetivo del presente estudio consiste en plasmar los valores predictivos de la mechanical power y el driving pressure. Especialmente como predictores de mortalidad. El modelo de investigación es una revisión de tipo documental bibliográfico. De la revisión se desprende que un PM superior a $17 \mathrm{~J} / \mathrm{min}$, se asocia con aumento en la mortalidad en la unidad de cuidados intensivos ( $\mathrm{UCI}$ ), mortalidad a los 30 días, menor número días sin ventilador y mayor duración de la estancia hospitalaria. Incluso con un bajo VC, un alto PM se asoció con mortalidad hospitalaria. Incluso, otros autores consideran que por encima de $12 \mathrm{~J} / \mathrm{min}$ se asocia con peores pronósticos y con síndrome de distrés respiratorio. Con respecto a la presión de distensión o conducción (driving pressure), los estudios han demostrado que, una DP mayor a $13 \mathrm{cmH} 2 \mathrm{O}$ y una presión meseta mayor a $24 \mathrm{cmH} 2 \mathrm{O}$ se asociaron directamente con una mortalidad mayor. En tal sentido, una mayor DP se asocia a una mayor probabilidad de mortalidad. Es fundamental el conocimiento y monitorización de las diferentes variables de la mecánica ventilatoria por cuanto representan una herramienta vital para el profesional de la salud en el manejo del paciente ventilado.

Palabras clave: Valor, Predictivo, Mortalidad, Poder mecánico, Presión de distensión.

\section{ABSTRACT}

Like any pharmacological intervention, mechanical ventilation (MV) must be titrated within a therapeutic window in order to achieve the objective for which it was initiated and at the same time limit the adverse effects of its use. It has been proposed that in "patients without acute respiratory distress syndrome (ARDS) who are subjected to mechanical ventilation, driving pressure and mechanical power are risk factors for an increase in mortality and the development of ARDS. The objective of this study is to capture the predictive values of mechanical power and driving pressure. Especially as predictors of mortality. The research model is a bibliographic documentary type review. The review shows that a MW greater than $17 \mathrm{~J} / \mathrm{min}$ is associated with increased mortality in the intensive care unit (ICU), mortality at 30 days, fewer days without a ventilator, and longer length of hospital stay. Even with a low CV, a high PM was associated with hospital mortality. Other authors even consider that above $12 \mathrm{~J} / \mathrm{min}$ is associated with worse prognoses and with respiratory distress syndrome. With regard to distension or driving pressure, studies have shown that a PD greater than $13 \mathrm{cmH} 2 \mathrm{O}$ and a plateau pressure greater than $24 \mathrm{cmH} 2 \mathrm{O}$ were directly associated with higher mortality. In this sense, a higher PD is associated with a higher probability of mortality. Knowledge and monitoring of the different variables of ventilatory mechanics is essential as they represent a vital tool for the health professional in the management of the ventilated patient.

Keywords: Value, Predictive, Mortality, Mechanical power, Distension pressure.

\section{RESUMO}

Como qualquer intervenção farmacológica, a ventilação mecânica (VM) deve ser titulada dentro de uma janela terapêutica a fim de atingir o objetivo para o qual foi iniciada e, ao mesmo tempo, limitar os efeitos adversos de seu uso. Tem sido proposto que em "pacientes sem síndrome do desconforto respiratório agudo (SDRA) que são submetidos à ventilação mecânica, a pressão de condução e a potência mecânica são fatores de risco para o aumento da mortalidade e o desenvolvimento da SDRA". O objetivo deste estudo é capturar os valores preditivos da potência mecânica e da pressão de condução. Especialmente como preditores de mortalidade. O modelo de pesquisa é uma revisão do tipo documentário bibliográfico. A revisão mostra que um MW maior que $17 \mathrm{~J} /$ min está associado ao aumento da mortalidade na unidade de terapia intensiva (UTI), mortalidade aos 30 dias, menos dias sem ventilador e maior tempo de internação hospitalar. Mesmo com um CV baixo, um PM alto foi associado com a mortalidade hospitalar. Outros autores até consideram que acima de $12 \mathrm{~J} /$ min está associado com pior prognóstico e com síndrome do desconforto respiratório. Com relação à distensão ou pressão de condução, estudos demonstraram que uma DP superior a $13 \mathrm{cmH} 2 \mathrm{O}$ e uma pressão de platô superior a $24 \mathrm{cmH} 2 \mathrm{O}$ estavam diretamente associadas a uma mortalidade mais elevada. Neste sentido, uma DP mais alta está associada a uma maior probabilidade de mortalidade. O conhecimento e o monitoramento das diferentes variáveis da mecânica ventilatória é essencial, pois elas representam uma ferramenta vital para o profissional de saúde no manejo do paciente ventilado.

Palavras-chave: Valor, Predictivo, Mortalidade, Poder mecânico, Pressão de distensão. 


\section{Introducción}

Tal y como resultaría con cualquier intervención farmacológica, la ventilación mecánica (VM) debe ser titulada dentro de una ventana terapéutica con el fin de que alcance el objetivo por el cual se inició y al mismo tiempo se limiten los efectos adversos de su empleo. (De Prost, Ricard, Saumon, \& Dreyfuss, 2011)

Las alteraciones pulmonares atribuibles a la VM han sido denominadas "lesión pulmonar asociada con la ventilación mecánica" (LPAVM). Rosas, Gutiérrez \& Cerón, (2017) refieren que, de forma clásica, se han descrito cuatro mecanismos de LPAVM los cuales son los siguientes: barotrauma, volutrauma, atelectotrauma y biotrauma. Más recientemente, se reconoció que la heterogeneidad regional, la frecuencia de estrés y la falla capilar pulmonar también contribuyen al desarrollo de LPAVM. (p. 320)

El desarrollo de lesión pulmonar asociada con la ventilación mecánica depende de la interacción de dos factores: uno de ellos es la cantidad de energía que entrega el ventilador, mientras que el otro depende de la respuesta del parénquima pulmonar a esa energía. Esta entrega de energía se conceptualiza en términos de poder mecánico, en donde se conjuntan variables que están asociadas al desarrollo de LPAVM, como el estrés, strain, flujo inspiratorio y frecuencia respiratoria. (Cressoni et al., 2016, p. 1101)

El Mechanical Power o poder mecánico, en física, determina la variable que indica la velocidad a la que se produce el trabajo, y se calcula teniendo en cuenta el trabajo desarrollado dividido por el tiempo que se emplea en realizarlo (J/min). En este contexto, la energía mecánica en fisiología pulmonar dependerá de la posición donde comienza el esfuerzo inspiratorio dentro de la curva presión-volumen del sistema respiratorio y de la fuerza impulsora ejercida por los músculos respiratorios para generar el movi- miento de la pared torácica. En los últimos años el concepto energía mecánica ha ganado nueva atención por la comunidad de cuidado intensivo cuando se reconoció que los parámetros del ventilador pueden interactuar con las fuerzas que actúan sobre la superficie pulmonar y contribuir a la lesión pulmonar inducida por ventilador. (Ortiz et al., 2020, p. 2, 3)

Por otra parte, el Driving Pressure (DP) o presión de distensión, fundamentalmente refleja el grado de estiramiento pulmonar durante un ciclo respiratorio. En ese contexto tiene relación con el strain dinámico; a mayor DP existe mayor strain dinámico del parénquima pulmonar y consecuentemente una mayor respuesta inflamatoria a nivel alveolar. Tanto el Vt (volumen corriente) como el PEEP (presión positiva al final de la espiración) "pueden modificar el driving pressure y generar mayor strain pulmonar dinámico y estático, respectivamente; no obstante, el daño pulmonar tiene mayor correlación con el strain dinámico que con el estático". (Montoya Zumaeta \& Cornejo Rosas, 2019, p. 2)

Por otra parte, debido a la dificultad para monitorizar la presión transpulmonar, se ha estudiado la presión de distensión como un subrogado de la presión transpulmonar. Análisis retrospectivos de grandes bases de datos obtenidas de ensayos clínicos aleatorizados han mostrado que la presión de distensión es el factor clave en la mortalidad. (Rosas, Gutiérrez, \& Cerón, 2017, p. 321)

Se ha propuesto que en "pacientes sin síndrome de distrés respiratorio agudo (SDRA) que son sometidos a ventilación mecánica, el driving pressure, la presión meseta inspiratoria y el poder mecánico son factores de riesgo para un aumento en la mortalidad y el desarrollo de SDRA". (Marín Martínez, 2019, p. 15) 
En virtud de lo expuesto, el paciente que requiere ventilación mecánica es un paciente de cuidado extremo por encontrase en un estado crítico, es decir, en riesgo de morir. La ventilación mecánica presenta un riesgo elevado de lesión pulmonar y mortalidad. En consecuencia, resulta imperioso que el personal de salud que atiende a este tipo de pacientes comprenda la tecnología y monitoreen los valores predictivos de las variables comprometidas en la ventilación mecánica con la finalidad de evaluar de manera certera su evolución o empeoramiento, y predecir su pronóstico o mortalidad.

En tal sentido, el objetivo del presente estudio consiste en plasmar los valores predictivos de la mechanical power y el driving pressure. Especialmente como predictores de mortalidad.

\section{Materiales y métodos}

La presente revisión fue desarrollada bajo el marco de una investigación de tipo documental bibliográfico. Para lo cual se ubicó material bibliográfico digitalizado que permitió desarrollar el objetivo planteado. Este material se ubicó mediante el uso de bases de datos tales como: PubMed, SciELO, Researchgate, Medigraphic, entre otras.

Los siguientes descriptores fueron los principales usados en la búsqueda y los que reflejaron mejores resultados: "Mechanical Power", "Driving pressure"; "Valor predictivo poder mecánico" y "Valor predictivo presión de distensión".

Con relación a la selección de los registros bibliográficos, se utilizaron criterios de filtrado tales como: idioma, el cual fue español e inglés, relevancia del tema y correlación temática. Igualmente, los trabajos seleccionaron tuvieron una fecha de publicación de 2011-2021. Es importante resaltar que la revisión fue realizada durante los meses de febrero - marzo del 2021.
La selección del material consistió en: libros digitalizados, e-books, protocolos, consensos, manuales, boletines informativos, ensayos clínicos, tesis de grado, posgrado o doctorado, informes, planes y proyectos, y otras clases de contenidos; asimismo guías de práctica clínica, estudios de cohorte y revisiones sistemáticas; que preferiblemente estuviesen producidos, avalados o promovidos por instituciones, entes, organizaciones, sociedades o asociaciones de profesionales en el área de la salud, que fueran de carácter público o privado, nacionales, internacionales o multilaterales. Fueron excluidos aquellos contenidos repetidos, las editoriales o cartas editoriales, anotaciones académicas y todo documento que no tuviera un sustento científico o bajo nivel de evidencia.

Los investigadores manifiestan no tener conflictos de intereses durante la elaboración del presente artículo.

\section{Resultados}

Es importante destacar que, recientemente tanto el mechanical power como la driving pressure, han sido propuestas como factores de riesgo para un aumento en la mortalidad y el desarrollo de SDRA, en pacientes sin síndrome de distrés respiratorio agudo (SDRA) que son sometidos a ventilación mecánica. En tal sentido, la monitorización de ambos valores, así como su control, en base a la evidencia científica, son de vital importancia para evitar el daño pulmonar que pueda producir la ventilación mecánica, así como sus consecuencias más fatales.

Power Mechanical o Poder Mecánico (PM) Gómez et al. citados por Marín Martínez, (2019) refieren que la lesión pulmonar inducida por el ventilador es generada por la interacción de distintos factores. Actualmente, se le ha prestado importancia a la cantidad de energía que entrega el ventilador al circuito respiratorio y se ha concep- 
tualizado en términos de poder mecánico conjuntando el estrés, la tensión, el flujo inspiratorio y la frecuencia respiratoria. Se ha definido como "la energía entregada por unidad de tiempo al sistema respiratorio por parte del ventilador mecánico y se mide en Joules/minuto. Se ha propuesto un valor de corte de 12 Joules/minuto ya que niveles superiores se han asociado al desarrollo de VILI (lesión pulmonar inducida por ventilador)". (p. 15)

Gattinoni et al., (2016) derivaron la fórmula del poder mecánico de la ecuación del movimiento, observando a través de estudios experimentales que un valor mayor de 12 Joules/minuto determina el desarrollo de LPAVM en sujetos sanos y empeoramiento clínico en pacientes con SDRA. La fórmula obtenida para el cálculo del PM es la siguiente:

\section{Poder Mecánico (PM): 0.098 x Vt x Fr x P pico -(Pplat-PEEP/2)}

Si los componentes de resistencia y la PEEP se suprimen del cálculo del PM, la influencia relacionada con la presión de conducción, $\triangle P(V T /$ distensibilidad) asumirá una relevancia aún mayor que su exponente al cuadrado $(\times 2)$ del volumen en las ecuaciones de energía y poder, y dado que la presión $(P)$ ha mostrado ser un predictor fuerte en la mortalidad en pacientes con SDRA, tiene mayor relevancia en el desarrollo de la VILI y debe enfatizarse que en conjunto, con la frecuencia con la que se repiten tales excursiones (ventilación minuto VE), podría ser un mejor indicador del riesgo de lesión pulmonar y se denomina «poder de conducción». (Marini \& Jaber, 2016)

Con respecto al Poder Mecánico y el peso corporal, Serpa Neto et al. citados por Ortiz et al., (2020) propusieron establecer lo siguiente:

Si el PM normalizado a tres índices antropométricos comunes (peso corporal predi- cho, índice de masa corporal - IMC y área de superficie corporal) eran mejores predictores de mortalidad hospitalaria que el PM absoluto en pacientes críticos bajo ventilación invasiva. Los resultados de este análisis post hoc se suman al primer informe sobre la normalización de PM, en donde concluyen que el PM normalizado al IMC tenía una asociación más fuerte con la mortalidad hospitalaria, dado que el tamaño absoluto del pulmón no aumentará con un incremento de la grasa corporal. Sin embargo, un IMC más alto puede aumentar el riesgo de atelectasia durante la ventilación y una disminución del tamaño funcional del pulmón. Por lo tanto, la normalización del PM al IMC puede reflejar mejor el PM entregado por unidad de tejido pulmonar ventilado, es decir, la «intensidad de ventilación». (p. 9)

Fuentes, Monares, Aguirre, \& Granillo, (2019) llevaron a cabo un estudio observacional en pacientes en ventilación mecánica invasiva ( $\mathrm{VMI}$ ), con una relación $\mathrm{PaO} 2 / \mathrm{FiO} 2$ $<200$ con al menos $5 \mathrm{cmH} 2 \mathrm{O}$ de PEEP. Se realizaron mediciones en los días 1 y 3 , utilizando las siguientes fórmulas de PM: Gattinoni $=0.098$ FR Vt (Ppico-1/2 $\triangle$ Paw); Marini $=$ 0.098 FR Vt Paw. Demostraron lo siguiente: La magnitud del PM de acuerdo con los parámetros iniciales no fue capaz de predecir el desenlace de mortalidad, pero al tercer día ambas fórmulas demostraron capacidad predictiva; ambas con una razón de riesgo ( $R R$ ) muy similar que se puede interpretar como una probabilidad de fallecer 05.65 veces más que quienes se mantuvieron por debajo del punto de corte de 22.4 $\mathrm{J} / \mathrm{min}$ y de $12 \mathrm{~J} / \mathrm{min}$ de las fórmulas del Dr. Gattinoni y del Dr. Marini, respectivamente. La significancia estadística se presentó con la fórmula del Dr. Gattinoni con p =0.02. Concluyeron que un PM elevado en la práctica clínica se asocia con mayor mortalidad en esta cohorte de pacientes en VMI prolongada (> 7 días). El PM es una herramienta prometedora que puede ser calculada a la cabecera del paciente. (p. 13, 14) 
Con el objetivo de determinar si el PM se asocia independientemente con resultados clínicos en pacientes críticos, se analizaron datos de dos cohortes, en las que se buscó examinar la asociación entre PM y mortalidad en pacientes con VM durante al menos 48 horas, encontrando que el PM superior a $17 \mathrm{~J} / \mathrm{min}$, se asocia con aumento en la mortalidad en la unidad de cuidados intensivos (UCI), mortalidad a los 30 días, menor número días sin ventilador y mayor duración de la estancia hospitalaria. Incluso con un bajo VC, un alto PM se asoció con mortalidad hospitalaria [OR 1,70 (1,32- 2,18)]; $\mathrm{p}<$ 0,001. (Ortiz Ruiz \& Dueñas Castell, 2021, p. 10)

Driving pressure o presión de conducción La presión de distensión (PD) o driving pressure (DP) "es una variable de la mecánica respiratoria que ha sido fuertemente asociada con mortalidad en pacientes con SDRA". (Pérez et al., 2018, p. 222)

Recientemente se ha propuesto al Driving Pressure como una herramienta de fácil acceso como un sustituto de la tensión dinámica durante la ventilación mecánica, siendo está definida como "La presión necesaria para sobrepasar el retroceso elástico del sistema respiratorio mientras el volumen corriente es inflado". Asimismo, se ha propuesto como valores de corte $>12-15$ cmH2O como indicadores de alta tensión pulmonar. Su cálculo se realiza fácilmente restando el PEEP a la presión meseta de la vía aérea:

$\triangle \mathrm{P}=$ Pmeseta $-\mathrm{PEEP}$ (Presión al final de la espiración). (Grieco, Chena, Dres, \& Brochard, 2017)

La DP es dependiente de la relación entre la distensibilidad del sistema respiratorio (CRS), la PEEP y el volumen corriente inspirado ( $\mathrm{Vt}$, volumen tidal), cuyos niveles elevados han demostrado impacto directo en el aumento de la mortalidad en pacientes con SDRA debido a la asociación que pre- senta con la lesión pulmonar inducida por la ventilación mecánica (VM). Loring et al. citados por Pérez et al, (2018), hacen énfasis en la "capacidad de la DP para predecir el pronóstico de los pacientes, dado que las variables que la definen (Vt, PEEP, Cst) son en sí mismas altamente predictivas para la supervivencia". (p. 223)

El grupo de trabajo LUNG SAFE realizó un estudio multicéntrico de tipo cohorte prospectivo incluyendo 50 países de cinco continentes, donde se midió la incidencia del SDRA, la capacidad para su reconocimiento clínico, la estrategia ventilatoria usada y otras terapéuticas adjuntas usadas para su tratamiento. El estudio "reportó en sus resultados que una DP mayor a $13 \mathrm{cmH} 2 \mathrm{O}$ y una presión meseta mayor a $24 \mathrm{cmH} 2 \mathrm{O}$ se asociaron directamente con una mortalidad mayor". Bellani et al., 2016)

Otros valores de DP asociados a mortalidad también han sido reportados: Chiumello, Carlesso, Brioni, \& Cressoni, (2016) los cuales estudiaron a 150 pacientes con SDRA y observaron que "los pacientes con DP mayor de $15 \mathrm{cmH} 2 \mathrm{O}$ presentaron mayor estrés alveolar y mayor elastancia pulmonar, concluyendo que una DP elevada puede relacionarse con el exceso de sobredistensión pulmonar con una precisión aceptable". ( $p$. 8)

Un estudio observacional y analítico de Villar et al. citado por Pérez et al., (2018) reportó que "niveles de DP mayores o iguales a $19 \mathrm{cmH} 2 \mathrm{O}$ se asocian a mayor mortalidad, misma que se relaciona con una presión meseta por arriba de $30 \mathrm{cmH} 2 \mathrm{O}^{\prime \prime}$. (p. 224)

Los avances tecnológicos han puesto a la disposición de la ciencia médica softwares programados en los ventiladores mecánicos capaces de realizar los cálculos necesarios para permitir la visualización continua de los valores (DP) en una pantalla, donde se encuentran programas además alarmas visuales y auditivas que señalan cuando se 
rebasan los niveles deseados de DP. Asimismo, pueden programarse otros rangos que permitan la protección pulmonar, como lo son el rango de Vt y la PEEP. (Pérez et al., 2018, p. 224)

A continuación, estos mismos autores presentan, a manera de ejemplo de esos softwares que permiten monitorear la función pulmonar para su protección, una tabla (Figura 1) de valores de presión de distensión (DP) de acuerdo con los niveles de distensibilidad del sistema respiratorio (CRS) medidos en el paciente y el volumen tidal $(\mathrm{Vt})$ y programado por el clínico. En el eje «X» de la tabla se muestran diferentes niveles de CRS medidos en el paciente bajo ventilación mecánica, en el eje «y» observamos diferentes niveles de Vt programados por el clínico, el resultado son los valores mostrados dentro de la tabla, obteniendo diferentes niveles de DP de acuerdo con la relación entre cada Vt programado con la CRS medida. En el área azul, denominada Zona de Seguridad, se encuentran niveles de DP menores de $13 \mathrm{cmH} 2 \mathrm{O}$ y en el área roja, denominada Zona de Riesgo, se encuentran niveles de DP mayores de $13 \mathrm{cmH} 2 \mathrm{O}$, los que se asocian a mayor mortalidad conforme aumenta su valor, todo esto en base al trabajo de Guérin et al.

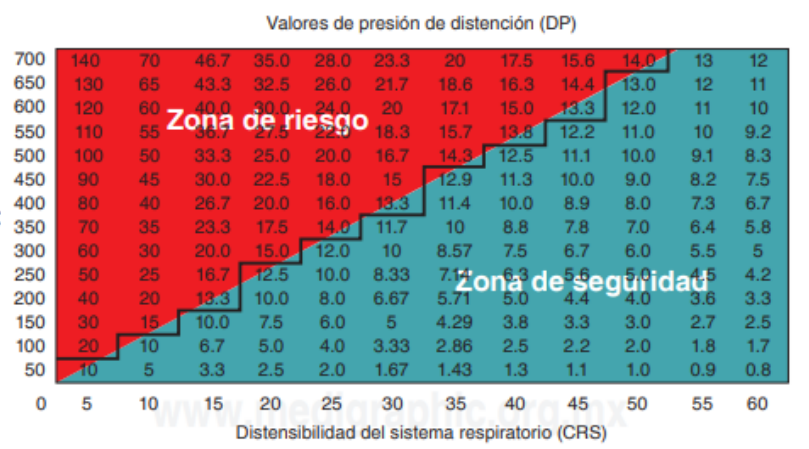

Figura 1. Tabla de valores de presión de distensión (DP) de acuerdo con los niveles de distensibilidad del sistema respiratorio (CRS) medidos en el paciente y el volumen tidal $(\mathrm{Vt})$ programado por el clínico. Recuperado de: "Presión de distensión (driving pressure): Principal objetivo para la protección alveolar". Pérez et al. (2018). Neumol Cir Torax. 77 (3). p. 225. URL: https://www.medigraphic.com/pdfs/ neumo/nt-2018/nt183j.pdf

\section{Conclusiones}

Es difícil traducir estudios experimentales en escenarios clínicos que permutan la predicción de la mortalidad hospitalaria, no obstante, este hecho sigue siendo objeto de investigaciones. En la práctica clínica diaria los parámetros ventilatorios son seleccionados y monitoreados, de manera individual, para cada paciente, tomando en consideración especialmente variables, tales como el poder mecánico y la presión de distensión o conducción, no obstante, no pueden evaluarse individualmente; si bien es cierto que son importantes predictores en la evolución e incluso de mortalidad del paciente, no es menos cierto que estas deben ser abordadas conjuntamente con el resto de las variables asociadas al proceso respiratorio.

De la revisión se desprende que un PM superior a $17 \mathrm{~J} / \mathrm{min}$, se asocia con aumento en la mortalidad en la unidad de cuidados intensivos (UCI), mortalidad a los 30 días, menor número días sin ventilador y mayor duración de la estancia hospitalaria. Incluso con un bajo VC, un alto PM se asoció con mortalidad hospitalaria. Incluso, otros autores consideran que por encima de $12 \mathrm{~J} / \mathrm{min}$ se asocia con peores pronósticos y con síndrome de distrés respiratorio.

Con respecto a la presión de distensión o conducción (driving pressure), los estudios han demostrado que, una DP mayor a 13 cmH2O y una presión meseta mayor a 24 $\mathrm{cmH} 2 \mathrm{O}$ se asociaron directamente con una mortalidad mayor. En tal sentido, una mayor DP se asocia a una mayor probabilidad de mortalidad.

Es fundamental el conocimiento y monitorización de las diferentes variables de la mecánica ventilatoria por cuanto representan una herramienta vital para el profesional de la salud en el manejo del paciente ventilado. 


\section{Bibliografía}

Bellani, G., Laffey, J. G., Pham, T., Fan, E., Brochard, L., Esteban, A., ... Ranieri, M. (2016). Epidemiología, patrones de atención y mortalidad de pacientes con síndrome de dificultad respiratoria aguda en unidades de cuidados intensivos en 50 países. JAMA, 315(8), 788-800. Recuperado el 15 de Marzo de 2021, de https://jamanetwork.com/journals/ jama/articlepdf/2492877/joi160008.pdf

Chiumello, D., Carlesso, E., Brioni, M., \& Cressoni, M. (2016). Presión de conducción de las vías respiratorias y estrés pulmonar en pacientes con SDRA. Crit Care, 20(276), 1-10. doi:10.1186/s13054-0161446-7

Cressoni, M., Gotti, M., Chiurazzi, C., Massari, D., Algieri, I., \& Amini, M. (2016). Potencia mecánica y desarrollo de lesión pulmonar inducida por ventilador. Anestesiología, 124(5), 1100-1108. Recuperado el 12 de Marzo de 2021, de https://pubs.asahq. org/anesthesiology/article/124/5/1100/14365/Mechanical-Power-and-Development-of-Ventilator

De Prost, N., Ricard, J. D., Saumon, G., \& Dreyfuss, D. (2011). Lesión pulmonar inducida por ventilador: perspectivas históricas e implicaciones clínicas. Ann Intensive Care, 28. Recuperado el 10 de Marzo de 2021, de https://annalsofintensivecare.springeropen.com/articles/10.1186/2110-5820-1-28

Fuentes, A. J., Monares, E., Aguirre, J. S., \& GraniIlo, J. (2019). El poder mecánico permite predecir mortalidad en pacientes en ventilación mecánica invasiva prolongada. Medicina Crítica, 33(1), 1014. Recuperado el 02 de Marzo de 2021, de https://www.medigraphic.com/pdfs/medcri/ti-2019/ ti191c.pdf

Gattinoni, L., Tonetti, T., Cressoni, M., Cadringher, P., Herrmann, P., Moerer, O., . . Quintel, M. (2016). Causas de lesión pulmonar relacionadas con el ventilador: la potencia mecánica. Intensive Care Med, 42(10), 1567-1575. Recuperado el 23 de Marzo de 2021, de https://link.springer.com/content/pdf/10.1007/s00134-016-4505-2.pdf

Grieco, D. L., Chena, L., Dres, M., \& Brochard, L. (2017). ¿Deberíamos utilizar la presión de conducción para volumen corriente? Curr Opin Crit Care, 23(0), 1-7. doi:10.1097/MCC.0000000000000377
Marín Martínez, A. D. (2019). Impacto de diferentes estrategias de ventilación mecánica sobre el driving pressure y el poder mecánico en pacientes sometidos a cirugía abdominal mayor bajo anestesia general. Tesis de post grado, Universidad Autónoma de Puebla, Facultad de Medicina, Puebla. Recuperado el 20 de Febrero de 2021, de https:// repositorioinstitucional.buap.mx/bitstream/handle/ 20.500.12371/10180/20200728130033-1602-T.pdf?sequence $=1$

Marini, J. J., \& Jaber, S. (2016). Dynamic predictors of VILI risk: beyond the driving pressure. Intensive Care Med, 42, 1597-1600. Recuperado el 02 de Marzo de 2021, de https://link.springer.com/content/pdf/10.1007/s00134-016-4534-x.pdf

Montoya Zumaeta, J., \& Cornejo Rosas, R. (2019). Driving Pressure, bases fisiológicas y aplicaciones clínicas. Revista Chilena de Medicina Intensiva, 34(3), 1-5. Recuperado el 25 de Marzo de 2021, de https://www.medicina-intensiva.cl/revista/pdf/68/8.pdf

Ortiz Ruiz, G., \& Dueñas Castell, C. R. (2021). Monitorización del paciente en ventilación mecánica. En G. Ortiz Ruiz, \& P. Cardinal Fernández, Experto en Patología Respiratoria Grave (pág. 11). Panamericana. Recuperado el 25 de Marzo de 2021, de http://aula.campuspanamericana.com/_Cursos/Curso01417/Temario/Experto_U_Patologia_ Respiratoria_Grave/M1T4texto.pdf

Ortiz, G., Cardinal, P., Dueña, C., Garay, M., Lara, A., \& Aguirre, Á. (2020). Poder mecánico. Acta Colomb Cuid Intensivo, 1-11. Recuperado el 21 de Febrero de 2021, de https://www.elsevier. es/es-revista-acta-colombiana-cuidado-intensivo-101-pdf-S0122726220300793

Pérez, O. R., Deloya, E., Lomelí, J. M., Pozos, K. P., Monares, E., \& Poblano, M. N. (2018). Presión de distensión (driving pressure): Principal objetivo para la protección alveolar. Neumol Cir Torax, 77(3), 222-227. Recuperado el 10 de Marzo de 2021, de https://www.medigraphic.com/pdfs/neumo/nt-2018/nt183j.pdf

Rosas, K., Gutiérrez, D., \& Cerón, U. W. (2017). Asociación y valor predictivo del poder mecánico con los días libres de ventilación mecánica. Medicina Crítica, 31(6), 320-325. Recuperado el 11 de Marzo de 2021, de http://www.scielo.org.mx/pdf/mccmmc/v31n6/2448-8909-mccmmc-31-06-320.pdf 


\section{CITAR ESTE ARTICULO:}

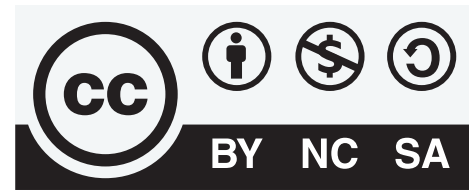

Choez Quimis, A. T., Quiroz Moncerrad, L. X., Hidalgo Bermúdez, C. A., \& González Plúa, C. L. (2021). Valor predictivo entre Mechanical Power y Driving Pressure. RECIMUNDO, 5(2), 119-127. https://doi.org/10.26820/recimundo/5.

(2).abril.2021.119-127 